Taulu S, Simola J, and Kajola M (2005). Applications of the Signal Space Separation Method. IEEE Trans Sign Proc 53, 3359-3372.

(C) 2005 IEEE. Reprinted with permission.

This material is posted here with permission of the IEEE. Such permission of the IEEE does not in any way imply IEEE endorsement of any of Helsinki University of Technology's products or services. Internal or personal use of this material is permitted. However, permission to reprint/republish this material for advertising or promotional purposes or for creating new collective works for resale or redistribution must be obtained from the IEEE by writing to pubs-permissions@ieee.org.

By choosing to view this document, you agree to all provisions of the copyright laws protecting it. 


\title{
Applications of the Signal Space Separation Method
}

\author{
Samu Taulu, Juha Simola, and Matti Kajola
}

\begin{abstract}
The reliability of biomagnetic measurements is traditionally challenged by external interference signals, movement artifacts, and comparison problems caused by different positions of the subjects or different sensor configurations. The Signal Space Separation method (SSS) idealizes magnetic multichannel signals by transforming them into device-independent idealized channels representing the measured data in uncorrelated form. The transformation has separate components for the biomagnetic and external interference signals, and thus, the biomagnetic signals can be reconstructed simply by leaving out the contribution of the external interference. The foundation of SSS is a basis spanning all multichannel signals of magnetic origin. It is based on Maxwell's equations and the geometry of the sensor array only, with the assumption that the sensors are located in a current free volume. SSS is demonstrated to provide suppression of external interference signals, standardization of different positions of the subject, standardization of different sensor configurations, compensation for distortions caused by movement of the subject (even a subject containing magnetic impurities), suppression of sporadic sensor artifacts, a tool for fine calibration of the device, extraction of biomagnetic DC fields, and an aid for realizing an active compensation system. Thus, SSS removes many limitations of traditional biomagnetic measurements.
\end{abstract}

Index Terms-Biomagnetism, calibration, DC measurements, interference suppression, magnetoencephalography, movement compensation, source modeling, spherical harmonics, virtual signals.

\section{INTRODUCTION}

B IOMAGNETIC measurements provide information of ionic current distributions in living organizms. For example, magnetoencephalography (MEG) [1] measures noninvasively the magnetic fields produced by the brain with a good spatial resolution and an excellent temporal resolution. These fields are very weak, and therefore, sensors with extreme sensitivity are required. Today, superconducting quantum interference devices (SQUIDs) [2] are the most widely used detectors of the biomagnetic fields, although recent developments with magnetoresistive elements [3] and optical magnetometers [4] may lead to practical biomagnetic applications. The SQUID sensors are typically operated in liquid helium $(4 \mathrm{~K})$, and they are located $2-4 \mathrm{~cm}$ from the skin. Modern measurement devices contain up to over 300 sensors.

The basic problem of biomagnetic measurements is the weakness of the signals, as compared with the external interference signals. In addition, the unprocessed MEG signals suffer from the fact that the coordinate systems of the head and the device are different. This complicates the comparison of different mea-

Manuscript received October 7, 2004; revised March 6, 2005. The associate editor coordinating the review of this manuscript and approving it for publication was Dr. Jan C. de Munck.

The authors are with Elekta Neuromag Oy, Helsinki, FI-00510 Finland (e-mail: Samu.Taulu@ neuromag.fi).

Digital Object Identifier 10.1109/TSP.2005.853302 surement sessions, even from the same subject, as the head usually cannot be fixed to the device. Furthermore, grand averages across different subjects may be biased because the subjects are not necessarily at the same position with respect to the device. Even more difficult problems are caused by movement of the subject during measurement because the movement distorts the biomagnetic field pattern and may also cause additional artifact signals if the subject has magnetic impurities, such as small magnetic particles, on the head or body.

Traditional methods for solving these problems deal with one problem at a time without attempting to find a general solution. These methods also often contain strong, artificial, and sometimes false assumptions of the spatial and temporal features of the signals sources.

The most common methods to reduce the effect of external interference signals are the magnetically shielded rooms [5]-[7] and gradiometer coils [1], [8]. To further suppress the residual interference inside the shielded room, reference channels [9] and signal space methods [10] have been widely used. Calculation of virtual signals and compensation for the movement distortions require the MEG signals to be represented with a device-independent source model. An example is the minimum-norm estimate (MNE) [11], [12] that has been used for movement correction [13] without separately modeling the external interference signals, as will be done in this paper. In transforming biomagnetic signals between different sensor configurations, MNE and multipole expansions of the field have been used [14], [15]. There have been no efficient solutions to deal with the movement artifacts caused by magnetic impurities. This significantly limits the applicability of MEG because several patient groups are prone to movement artifacts, sometimes rendering the data useless for further analysis.

Because of the lack of robust general-purpose methods to improve the quality of MEG data, experimenters usually try to minimize the effect of signal distortions in the raw MEG signals. This requires extreme magnetic hygiene inside the shielded room, minimization of the movement, and sometimes even demagnetization of the subjects. Therefore, traditional MEG measurements require very careful preparations, highly trained personnel, and special, nonmagnetic equipment. However, even with careful preparation, it is impossible, especially in the clinic but also in the research laboratory, to suppress all distortions from MEG data with these precautions.

In this paper, we show how the multichannel MEG signals can be transformed into an idealized form that is free of the problems mentioned above, thus relaxing the strict limitations and requirements related to conventional MEG measurements. The idealization is done by the signal space separation method (SSS) [16], [17] that transforms the data collected with a high number of channels into idealized signals containing uncorrelated information of 
the underlying current distributions. SSS is based only on exact knowledge on the sensor geometry, Maxwell's equations, and the quasistatic approximation. Furthermore, we rely on the fact that all sources of magnetic fields (both biomagnetic and external interference sources) are located more than a couple of centimeters away from the sensors. It will be shown that SSS is able to suppress external interference signals, produce virtual signals, and compensate for movement distortions and artifacts with one linear transformation. In SSS, the only necessary a priori information is the knowledge of the geometry of the sensor array and its relative position with respect to the head.

The purpose of this paper is to give a comprehensive but less formal description of SSS than that presented in our theoretical paper [17]. Furthermore, we will review some of the most common problems of biomagnetic measurements and demonstrate with practical examples how SSS solves these problems.

\section{IDEALIZATION OF MEASURED SignALS}

\section{A. Spatial Filtering and the SSS Basis}

A modern MEG device records the neuromagnetic field distribution by sampling the field simultaneously at 200-500 distinct locations around the subject's head. When the total number of recording channels is $N$, the measurement result at each moment of time is a vector $\phi$, comprising $N$ components. The direction of the signal vector in the $N$-dimensional signal space is determined by the ratios of the vector components, that is, the ratios of the signal amplitudes in the different channels. In principle, any direction in the signal space represents a valid result of a magnetic field measurement. However, by combining what is known about the location of possible sources of magnetic field, the geometry of the MEG sensor array, and some basic electromagnetic theory, it is possible to differentiate between the signal space directions that are meaningful results of a neuromagnetic recording and those that are not and, thus, considerably constrain the relevant signal space.

The field distribution recorded by an MEG sensor array arises from sources outside of the volume where the sensors themselves are located. This is because the MEG devices, to avoid instrument artifacts, are constructed so that the sensors and their immediate vicinity are free of magnetic sources and materials. In this case, the recorded field is a gradient of a scalar potential that is free of singularities and harmonic in the volume containing the sensors [18].

A harmonic potential $V(\mathbf{r})$ is a solution of the Laplacian differential equation $\nabla^{2} V=0$. Several different sets of harmonic functions have been presented in the mathematical literature during the last 150 years. Spherical harmonic functions, which are usually applied with spherical coordinate system, are an example of such a set of harmonic functions. These functions form a complete set, meaning that any harmonic function in a three-dimensional space can be presented with arbitrary accuracy as a series expansion of these functions. Therefore, we can express any harmonic potential $V(\mathbf{r})$ as an expansion

$$
V(\mathbf{r})=\sum_{l=1}^{\infty} \sum_{m=-l}^{l} \alpha_{l m} \frac{Y_{l m}(\theta, \varphi)}{r^{l+1}}+\sum_{l=1}^{\infty} \sum_{m=-l}^{l} \beta_{l m} r^{l} Y_{l m}(\theta, \varphi)
$$

where

$$
Y_{l m}(\theta, \varphi)=\sqrt{\frac{2 l+1}{4 \pi} \frac{(l-m) !}{(l+m) !}} P_{l m}(\cos \theta) \mathrm{e}^{\mathrm{i} m \varphi}
$$

is the normalized spherical harmonic function, $r, \theta$, and $\varphi$ are the spherical coordinates, $P_{l m}(\cos \theta)$ is the associated Legendre function, and i denotes imaginary unit. In practice, $V(\mathbf{r})$ is realvalued, although the calculations can be done compactly with complex numbers. A direct real-valued expansion for the potential is given, e.g., in [19].

The spherical harmonic functions are labeled with two indices $l$ and $m$, with $l$ running from 0 to infinity and $m$ running from $-l$ to $l$. The potential with $l=0, m=0$ is associated with the field of a magnetic monopole and is therefore excluded from the expansion. When going to higher index values, these functions contain increasingly higher spatial frequencies. The radial $r$-dependent part of the expansion separates into two sets of functions: Those proportional to inverse powers of $r$ are singular at the origin, and those proportional to powers of $r$ diverge at infinity.

Given an array of MEG sensors and a coordinate system with its origin somewhere inside of the helmet, we can calculate the signal vectors corresponding to each of the terms in (1). Let us denote the signal vector corresponding to term $Y_{l m}(\theta, \varphi) / r^{l+1}$ as $\mathbf{a}_{l m}$ and, similarly, the signal vector corresponding to term ${ }^{l} Y_{l m}(\theta, \varphi)$ as $\mathbf{b}_{l m}$. A set of $N$ such signal vectors forms a basis in the $N$-dimensional signal space [17], and hence, the signal vector is given as

$$
\phi=\sum_{l=1}^{\infty} \sum_{m=-l}^{l} \alpha_{l m} \mathbf{a}_{l m}+\sum_{l=1}^{\infty} \sum_{m=-l}^{l} \beta_{l m} \mathbf{b}_{l m}
$$

This basis is not orthogonal, but it is linearly independent so that any measured signal vector has a unique coordinate presentation $\mathbf{x}$ in this basis. The signal space separation method is based on this coordinate presentation known as the " $l, m$-spectrum" of the recorded signal. Mathematically, this can be expressed as

$$
\phi=\mathbf{S x}=\left[\mathbf{S}_{\text {in }} \mathbf{S}_{\text {out }}\right]\left[\begin{array}{c}
\mathbf{x}_{\text {in }} \\
\mathbf{x}_{\text {out }}
\end{array}\right]
$$

where the sub-bases $\mathbf{S}_{\text {in }}$ and $\mathbf{S}_{\text {out }}$ contain the basis vectors $\mathbf{a}_{l m}$ and $\mathbf{b}_{l m}$, respectively, and vectors $\mathbf{x}_{\mathrm{in}}$ and $\mathbf{x}_{\text {out }}$ contain the corresponding $\alpha_{l m}$ and $\beta_{l m}$ values (the $l, m$ spectra), respectively.

Utilizing this coordinate presentation, we can first do spatial filtering in a very meaningful way. The signals from real magnetic sources, located at distances larger than about $2 \mathrm{~cm}$ from the sensors, are mostly contained in the low $l, m$ end of the spectrum. Inherent instrument noise and artifacts of single channels, being totally uncorrelated spatially, are evenly distributed among all of the spectral components. Therefore, we may filter the measured data by including the low $l, m$ components up to the limit where the contribution arising from the real sources becomes immeasurably small because it is buried in the sensor noise that dominates the highest $l, m$ components. This process of leaving out the high $l, m$ end of the spectrum not only reduces the noise like ordinary spatial filtering but also guarantees that 
the retained field distribution is consistent with Maxwell's equations $\nabla \cdot \mathbf{b}=0$ and the quasistatic $\nabla \times \mathbf{b}=\mathbf{0}$ in empty space. Therefore, we call this procedure "Maxwell filtering."

Additionally, based on this coordinate presentation, we can perform signal space separation, that is, separate the components of field arising from sources inside and outside of the helmet, respectively. It can be shown that the basis vectors, corresponding to the terms in the second sum in expansion (1), represent the sources external to the helmet [17]. By leaving those components of the recorded signal out from the reconstructed coordinates $\mathbf{x}$, we are left with the part of the signal coming from inside of the helmet only. This part of the signal vector consists of the spectral components corresponding to terms diverging at the origin, i.e., the terms in the first sum in (1).

This separation into two independent sub-bases fails only in the in-practice highly unlikely case when all of the sensors are on the surface of a sphere, strictly radial, or strictly tangential, and the coordinate origin is placed in the center of this sphere [17]. In this case, the basis vectors obtained with same $l$ and $m$ but corresponding to the radially converging and diverging terms, respectively, are identical.

With real data measured with an Elekta Neuromag ${ }^{\circledR}$ 306channel device, it turns out that including the components up to $l=L_{\text {in }}=8$ for the $S_{\text {in }}$ basis, and up to $l=L_{\text {out }}=4$ for the $\mathbf{S}_{\text {out }}$ basis is optimal in most cases for both the Maxwell filtering and signal source separation operations. The sufficiency of this choice of $L_{\text {in }}$ is demonstrated by simulation results shown in Figs. 1 and 2, which demonstrate the ability of basis $\mathbf{S}_{\text {in }}$ to model signals produced by sources with varying spatial extent. Fig. 1 shows the subspace angle between $\mathbf{S}_{\text {in }}$ and the signal vector produced by a single dipole as a function of distance of the dipole from the origin of the harmonic expansion with values $L_{\text {in }}=2$ to 9 . This angle should be compared with the signal-to-noise ratio (SNR), which here is defined as the ratio of the norms of the 306-channel signal vector of Elekta Neuromag ${ }^{\circledR}$ and the corresponding noise vector. As a randomly chosen noise vector in 306-dimensional space is approximately orthogonal to the signal vector, the angle between the measured and the actual noiseless signal vector is about $\arctan (1 / \mathrm{SNR})$.

Fig. 2 is similar to Fig. 1, but in this case, the source consists of 100 simultaneously active dipoles distributed on the surface of a sphere. The subspace angle is shown as a function of the radius of the sphere. The comparison of Figs. 1 and 2 indicates that the performance of SSS is essentially independent of the focality of the source. The practical multichannel sensor arrays with finite noise level are not capable of extracting all the details of extremely complex spatial source patterns, and SSS covers, with $L_{\text {in }}=8$, all measurable features of the field, irrespective of the complexity of the source distribution. The figures give both the subspace angle and the corresponding SNR values. It can be seen that for a typical superficial source distance of $7 \mathrm{~cm}$, corresponding to distances of about $3.5 \mathrm{~cm}$ from the sensors to the closest dipoles, the effect of SSS on signal morphology only shows, with considerably large SNR, values higher than 30 , even for a complex source composed of 100 dipoles.

In practice, the choice of $L_{\text {out }}$ affects the reconstruction error if $L_{\text {in }}$ is not high enough for $\mathbf{S}_{\text {in }}$ to completely model the signals arising from internal sources. In such a case, part of the energy

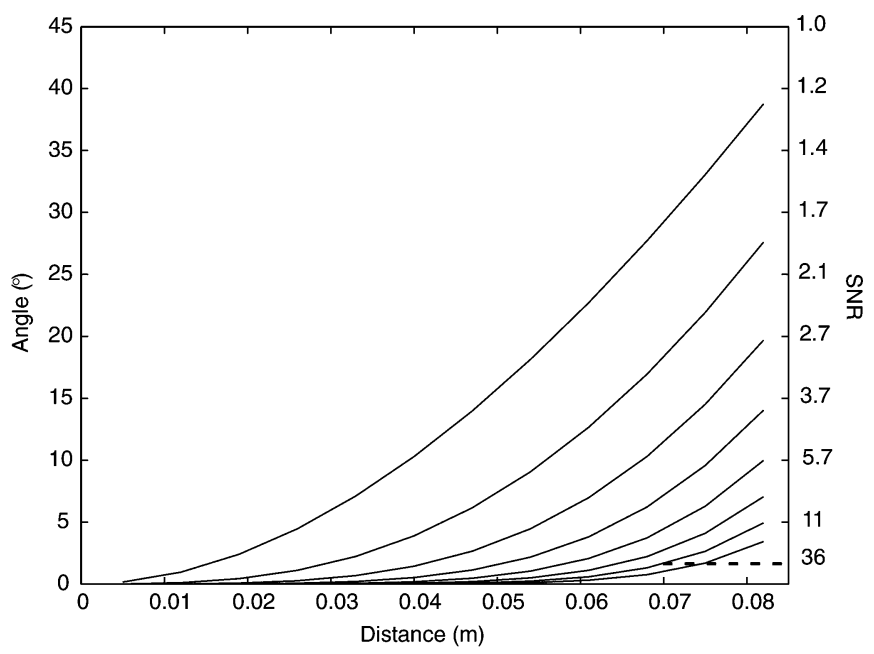

Fig. 1. Angle between $\mathbf{S}_{\text {in }}$ and the signal vector produced by a single current dipole as a function of distance of the dipole from the origin of the harmonic expansion (center of the device coordinate system) with values $L_{\text {in }}=2$ to 9 . The curves are in descending order, starting from the lowest values of $L_{\text {in }}$. The theoretical SNR values corresponding to the given subspace angle are given on the right-hand side.

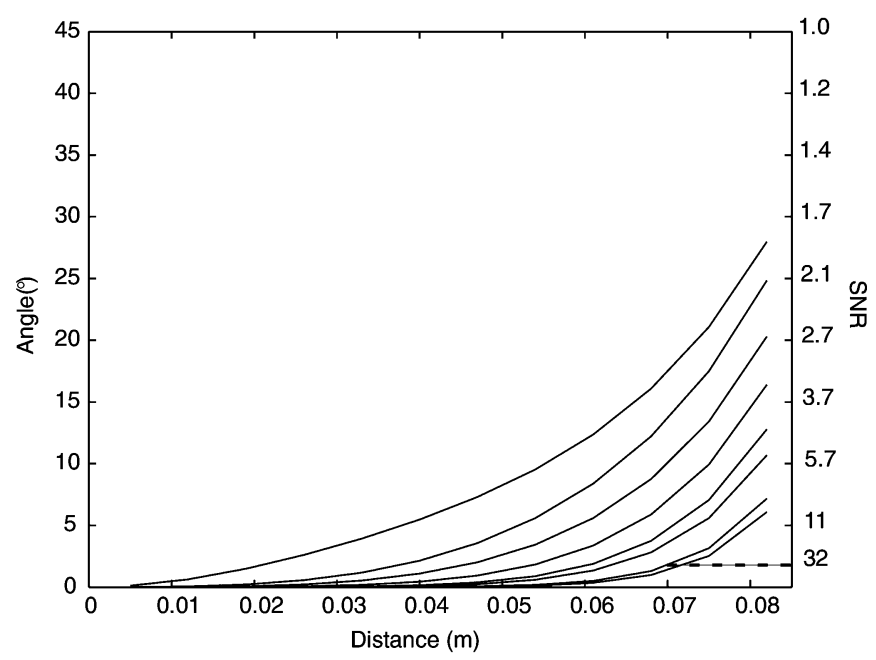

Fig. 2. Angle between $\mathbf{S}_{\text {in }}$ and the signal vector produced by a combination of 100 current dipoles on a sphere as a function of the radius of the sphere with values $L_{\text {in }}=2$ to 9 . The curves are in descending order, starting from the lowest values of $L_{\text {in }}$. The theoretical SNR values corresponding to the given subspace angle are given on the right-hand side.

of the internal signals falls into $\mathbf{S}_{\text {out }}$ in the SSS reconstruction. To demonstrate the effect, Fig. 3 shows the angle between the dipole signal vector used in Fig. 1 and the reconstructed signal vector produced by decomposing the signal in the SSS basis and reconstructing the signal from components corresponding to $\mathbf{S}_{\text {in }}$ only. It can be seen that with high enough $L_{\text {in }}$, the reconstruction error does not essentially differ from that shown in Fig. 1.

The small change of signal morphology can be taken into account in the source modeling of e.g., a current dipole in such a way that no localization bias results. However, even if the truncation to the finite $L_{\text {in }}$ is not properly taken into account, the resulting bias is negligible when compared with other sources of localization error. This is demonstrated in Fig. 4, where the localization error of a randomly chosen dipole is shown as a function of $L_{\text {in }}$ after decomposing the signal vector of the dipole to 


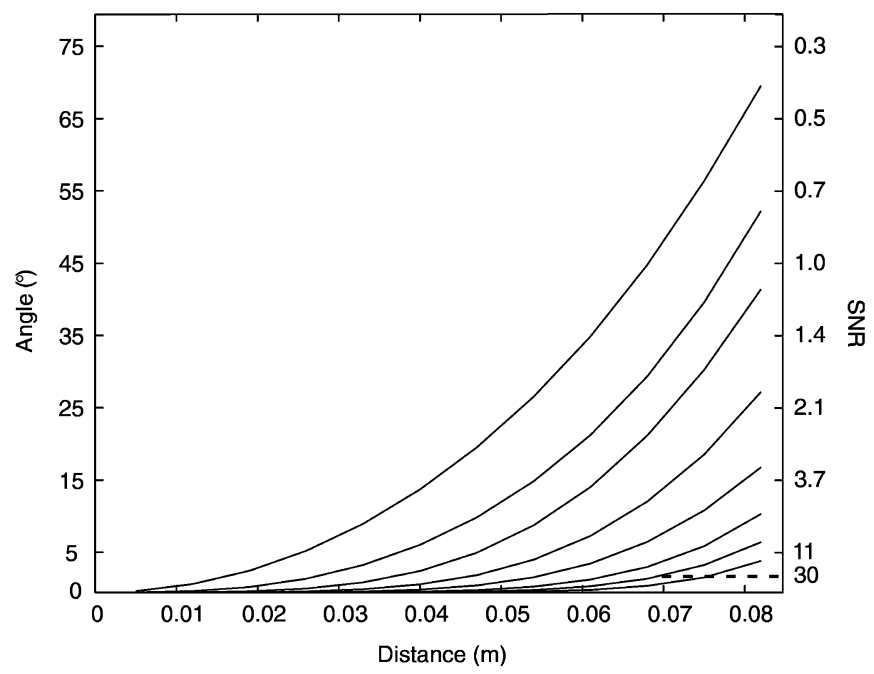

Fig. 3. Angle between the signal vector produced by a single current dipole and the corresponding SSS reconstructed signal vector as a function of distance of the dipole from the origin of the harmonic expansion (center of the device coordinate system) with values $L_{\text {in }}=2$ to 9 and $L_{\text {out }}=3$. The curves are in descending order, starting from the lowest values of $L_{\text {in }}$. The theoretical SNR values corresponding to the given subspace angle are given on the right-hand side.

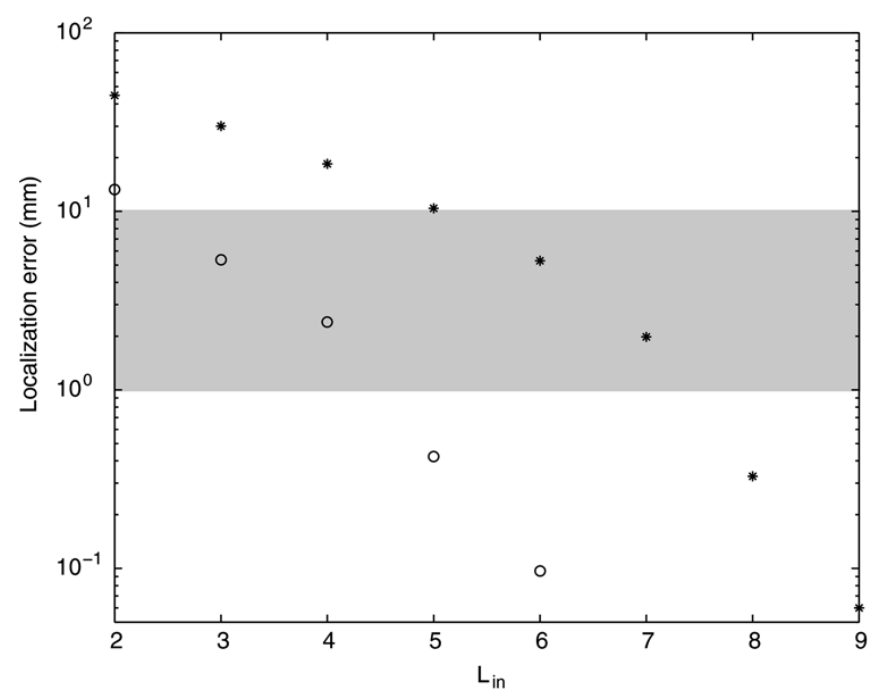

Fig. 4. Localization error from an SSS reconstructed dipole signal as a function of $L_{\text {in }}$ with $L_{\text {out }}=4$. The circles and stars correspond to a dipole with distance of 4 and $7 \mathrm{~cm}$ from the origin of the expansion, respectively. The gray bar indicates the range of the linear confidence dimension of dipole localization between typical high- and low-quality data.

SSS basis with the given $L_{\mathrm{in}}$ and $L_{\mathrm{out}}=4$ and reconstructing the signal from components corresponding to $\mathbf{S}_{\text {in }}$ only. The errors are shown for a deep and a superficial dipole located at distances of 4 and $7 \mathrm{~cm}$ from the origin, respectively. It can be seen that the localization error imposed by SSS becomes insignificantly small with $L_{\text {in }} \geq 5$ for the deep dipole and $L_{\text {in }} \geq 8$ for the superficial dipole, as compared to $1 \mathrm{~mm}$, which is the localization accuracy of even the highest quality MEG data.

The simulations used the spherical conductor model in the forward calculation of the dipole signal but are also applicable for a realistic head because SSS is independent of conductor models.

In the most demanding applications of the SSS method, both $L_{\mathrm{in}}$ and $L_{\mathrm{out}}$, as well as the location of the coordinate origin, can be used as freely adjustable parameters. In some extreme cases, like a small baby's heart being a source of large amplitude interference in the immediate vicinity of the helmet, $L_{\text {out }}=6$ may be required. The only limitation as to the choice of $L_{\text {in }}$ and $L_{\text {out }}$ is that the total number of basis vectors $n=\left(L_{\text {in }}+1\right)^{2}+$ $\left(L_{\text {out }}+1\right)^{2}-2$ must be smaller than or, at most, equal to the dimension of the signal space, that is, the total number channels in the MEG device.

\section{B. Effect of SSS Reconstruction on Signals and Noise}

The effect of the SSS process on the signals, interference, and noise in the SSS process is schematically illustrated in Fig. 5. For clarity, the signal space is here reduced to two dimensions: one containing the entire $\mathbf{S}_{\text {in }}$ basis and the other containing the entire $\mathbf{S}_{\text {out }}$ basis. The SSS basis is not orthogonal, and therefore, the angle $\psi$ between the "interference space" $\mathbf{S}_{\text {out }}$ and the "brain signal space" $\mathbf{S}_{\text {in }}$ is not $90^{\circ}$. The signal vector $\boldsymbol{\phi}$ is decomposed into two components $\phi_{\text {out }}$ and $\phi_{\text {in }}$. The latter component faithfully reproduces, in all the MEG channels, the signals that would be seen if the interference from the sources external to the helmet were absent.

The signal decomposition by SSS is different from the software gradiometrization method, or Signal Space Projection method (SSP) [10], both of which are projection methods that are commonly used to remove interference from recorded MEG data. In these methods, the interference space $\mathbf{S}_{\text {out }}$ is either defined to consist of field gradients up to a given order or, in SSP, determined by a statistical analysis of an empty room recording made without a subject. Subsequently, all recorded data is projected on the subspace orthogonal to $\mathbf{S}_{\text {out }}$ giving the estimate $\phi_{\perp}$ for the brain signal. This removes the interference but, as seen by comparing $\phi_{\text {in }}$ and $\phi_{\perp}$ from Fig. 5, also leads to removal of the brain signal components falling on the $\mathbf{S}_{\text {out }}$ space and, thus, to a distortion of the actual brain signal. In source modeling, this distortion must, of course, be taken into account properly so that no localization bias results.

In addition, the qualitative effects of the SSS and the projection operations on the noise are different and can be concluded from Fig. 5. There is no increase of noise associated with the projection methods. Their effect is rather to slightly decrease the noise because the projection decreases the signal dimensionality: Less than $N$ output quantities are derived out of $N$ measurements. In the SSS method, there are two counteracting mechanisms affecting the noise: The Maxwell filtering reduces the noise because, like in the projection operations, the dimensionality is reduced when leaving out the high $l, m$ components. On the other hand, as can be seen from Fig. 5, the noise is enhanced when the signal vector is decomposed in an oblique basis where the angle between the $\mathbf{S}_{\text {in }}$ and $\mathbf{S}_{\text {out }}$ subspaces $\psi$ is less than $90^{\circ}$.

This angle, however, can be effectively influenced by proper design of the sensor array. A larger angle $\psi$ is obtained by an array design that incorporates both magnetometers and gradiometers compared with a design with only one type of sensors. This is because an array containing both gradiometers and magnetometers has a built-in tendency to resolve between signals coming from nearby and far-away sources. The basis 


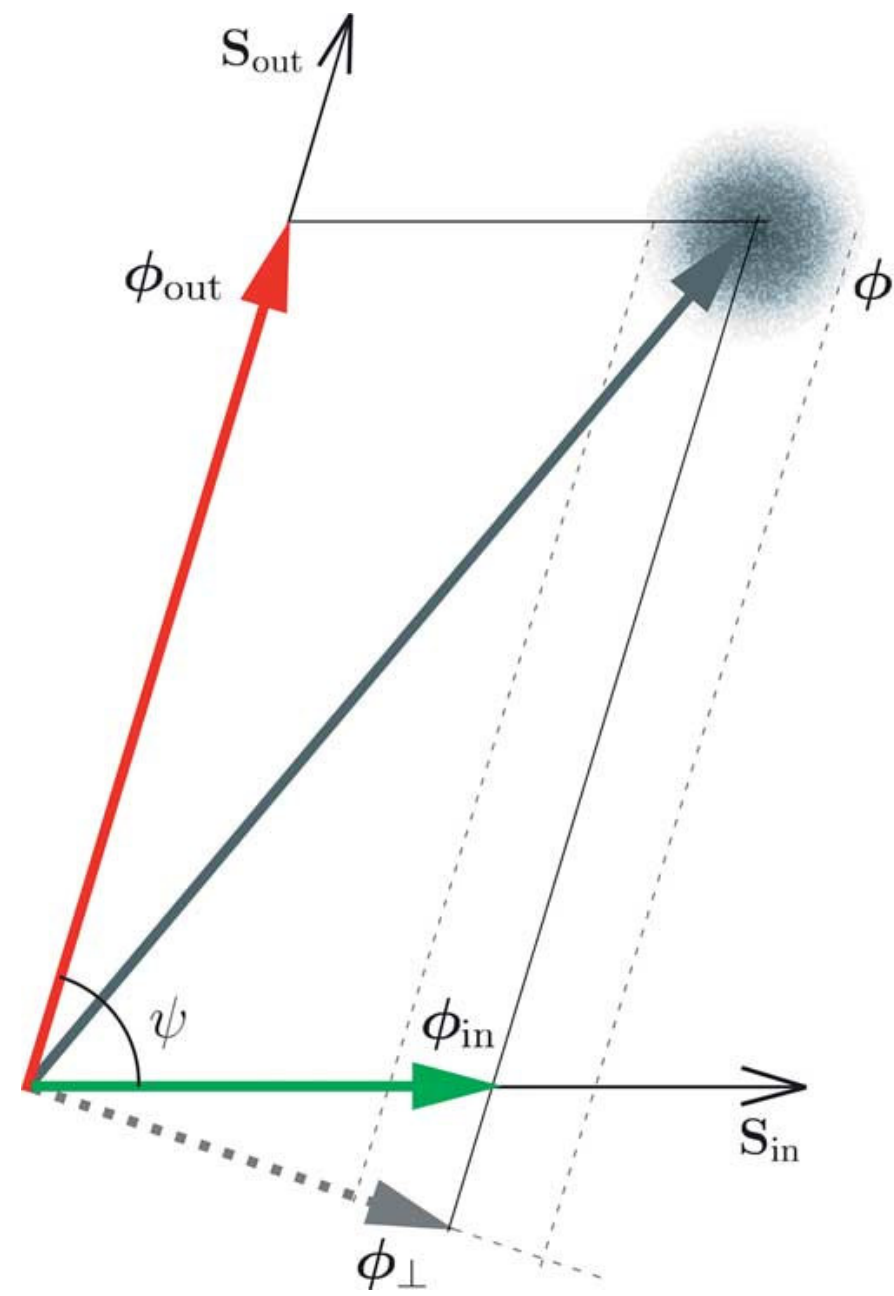

Fig. 5. Graphical illustration of the signal space separation method. The measured signal vector $\phi$ is decomposed into two components $\phi_{\text {in }}$ and $\phi_{\text {out }}$ The $\phi_{\text {in }}$ component reproduces the biomagnetic signal undistorted, whereas the projected component $\phi_{\perp}$ resulting from interference suppression by software gradiometrization, or by signal space projection, shows a change $\phi_{\perp}-\phi_{\text {in }}$ in signal morphology. The gray sphere indicates random noise.

vectors contained in the $\mathbf{S}_{\text {out }}$ basis for such an array are predominantly along the magnetometer dimensions of the signal space, whereas in the $\mathbf{S}_{\text {in }}$ basis, the gradiometric dimensions dominate. This partial signal separation already taking place on the hardware level results in a small condition number of the SSS basis, which means a large angle $\psi$. The condition number of a matrix is defined as the ratio of the largest and smallest singular values of the matrix.

In principle, axial gradiometers are as good as planar gradiometers for stabilizing the SSS basis of a multichannel MEG device. Technically, however, planar gradiometers have two advantages over axial gradiometers. First, they are easy to overlap with magnetometers and each other, which ensures large pick-up area and, thus, high sensitivity of individual channels, even if the total number of channels exceeds 300 . All existing axial systems are based on nonoverlapping pick-up coils, which is a solution that leads to reduction of pick-up area and sensitivity of individual channels when the number of channels already exceeds 200. Second, an accurate geometric parametrization and fine calibration-a prerequisite for efficient use of SSS - is easy for the magnetometer/planar gra-
TABLE I

Minimum, MEAN, AND MAXIMUM PRINCIPAL ANGLES (IN DEGREES) FOR THREE DEVICES AS A FUNCTION OF $L_{\text {Out }}$

\begin{tabular}{c||c||c||c}
\hline & 248 -ch system & 275 -ch system & 306 -ch system \\
\hline$L_{\text {out }}=2$ & $2.8 / 4.0 / 5.5$ & $2.7 / 3.8 / 5.0$ & $5.7 / 10.1 / 13.4$ \\
$L_{\text {out }}=3$ & $2.4 / 4.8 / 10.0$ & $1.7 / 4.6 / 8.5$ & $5.0 / 10.8 / 24.7$ \\
$L_{\text {out }}=4$ & $2.0 / 7.1 / 32.2$ & $1.4 / 6.6 / 19.0$ & $2.3 / 13.6 / 64.3$ \\
$L_{\text {out }}=5$ & $1.5 / 9.9 / 48.2$ & $1.2 / 9.7 / 43.1$ & $1.0 / 16.4 / 73.2$ \\
\hline
\end{tabular}

diometer combination because these sensors can be fabricated, for example, with a precise thin film process on a single planar silicon surface.

In practice, $\psi$ represents the principal angles between subspaces $\mathbf{S}_{\text {in }}$ and $\mathbf{S}_{\text {out }}$. The largest principal angle is commonly used as the subspace angle, and its sine is defined as the distance between two equidimensional subspaces [20]. However, the whole spectrum of the angles plays a role in the SSS reconstruction, and thus, the largest principal angle cannot be taken as a unique value for $\psi$. To get an idea of the performance of different sensor arrays, Table I shows the smallest, mean, and the largest principal angle as a function of $L_{\text {out }}$ for a 248-channel magnetometer system, a 275-channel system containing axial gradiometers, and a 306-channel system containing 204 gradiometers and 102 magnetometers with two orthogonal planar gradiometers and one magnetometer on each sensor element. In this calculation, $L_{\text {in }}=8$ was used.

The relation between noise and the principal angles is not trivial. To get a more quantitative view on the noise performance of the different systems, we simulated the effect of the SSS reconstruction on the noise levels of the sensor arrays used in Table I. In the simulation, SSS reconstruction was performed on signals consisiting of normal distributed random noise with equal standard deviation for all channels. It turns out that, on average, with $L_{\text {in }}=8$ and $L_{\text {out }}=3$, the random noise is increased by factors 3.7 and 3.6 for the hypothetic 248- and 275-channel systems, respectively. In the case of the 306-channel system, the magnetometer noise is increased by a factor of 2.4 , but the gradiometer noise is decreased, having an average relative noise of 0.61. In reality, however, the effect of SSS on the noise of MEG data is not as significant as this simulation indicates because here, we only take into account the sensor noise, whereas real noise also contains contributions from the brain and the radiation shields. The brain signal component is left intact in the SSS reconstruction as it represents sources inside the sensor array. The dewar noise component represents sources in the immediate vicinity of the sensors and is, thus, not completely included in the SSS model but is still not significantly modified by SSS. This is also consistent with our experimental observations: We have not found the SSS reconstruction to essentially change the noise levels, even those of the magnetometers, in the 306-channel MEG recordings.

\section{Idealized Channels}

We define the harmonic amplitudes $\alpha_{l m}$ as idealized channels; see (1). They correspond to the biomagnetic signals only 
and are free of movement distortions as they are attached to the coordinate system of the head. Furthermore, they contain the information of the underlying current distribution $\mathbf{J}(\mathbf{r})$ in a more compact form than the raw signals from the real channels. This is easily seen by comparing the lead field forms of the idealized and real channels. For the definition of the lead field, see, e.g., [1], where the lead field of an individual sensor has been defined for primary current by taking the conductivity distribution into account to correctly incorporate the effect of the volume currents. However, the accurate conductivity distribution of the biomagnetic current volume is generally unknown, and here, we want to show that the idealized channels provide uncorrelated information of the general current distribution located inside the sensor array, regardless of the conductivity patterns. Therefore, we examine the total current, which is a sum of the primary and volume currents.

In [17], the lead field of the idealized channel $\alpha_{l m}$ is given as

$$
\lambda_{l m}^{\alpha}(\mathbf{r})=\frac{\mathrm{i}}{2 l+1} \sqrt{\frac{l}{l+1}} r^{l} \mathbf{X}_{l m}^{*}(\theta, \varphi)
$$

where $\mathbf{X}_{l m}(\theta, \varphi)$ is the vector spherical harmonic function [18], [21], [22]. On the other hand, by inserting this lead field to the expression for the magnetic field [17], one gets the following expression for the magnetic flux through an individual sensor:

$$
\begin{aligned}
\phi_{i}= & \int_{A_{i}} \mathbf{B}(\mathbf{r}) \cdot d \mathbf{A}_{i} \\
= & -\mu_{0} \int_{v^{\prime}}\left[\sum_{l=1}^{\infty} \sum_{m=-l}^{l} \lambda_{l m}^{\alpha}\left(\mathbf{r}^{\prime}\right)\right. \\
& \left.\times \int_{A_{i}} \frac{\boldsymbol{\nu}_{l m}(\theta, \varphi)}{r^{l+2}} \cdot d \mathbf{A}_{i}\right] \cdot \mathbf{J}\left(\mathbf{r}^{\prime}\right) d v^{\prime}
\end{aligned}
$$

where $\mu_{0}$ is the permeability of a vacuum, $d \mathbf{A}_{i}$ is the surface element of the sensor $i$, and $\boldsymbol{\nu}_{l m}(\theta, \varphi)$ is the modified vector spherical harmonic function defined in [17]. The integration volume $v^{\prime}$ extends over a spherical volume that fits into the volume inside of the sensor array and encloses the biomagnetic currents. Thus, the lead field $\mathbf{L}_{i}$ of an individual sensor for the total current is a linear combination of the lead fields of the idealized channels

$$
\mathbf{L}_{i}\left(\mathbf{r}^{\prime}\right)=-\mu_{0} \sum_{l=1}^{\infty} \sum_{m=-l}^{l} \lambda_{l m}^{\alpha}\left(\mathbf{r}^{\prime}\right) \int_{A_{i}} \frac{\nu_{l m}(\theta, \varphi)}{r^{l+2}} \cdot d \mathbf{A}_{i} .
$$

Because of the orthonormality of the vector spherical harmonic functions $\mathbf{X}_{l m}(\theta, \varphi)$, the lead fields $\lambda_{l m}^{\alpha}(\mathbf{r})$ of the idealized channels $\alpha_{l m}$ are orthogonal. Thus, the idealized channels contain uncorrelated information of the underlying current distribution. In contrast, the lead fields $\mathbf{L}_{i}\left(\mathbf{r}^{\prime}\right)$ of the real channels are not orthogonal; see (7).

The idealized channels are the components of vector $\mathbf{x}_{\text {in }}$ in (4), and they can be estimated from the measured signal vector $\phi$ as

$$
\hat{\mathbf{x}}=\left[\begin{array}{c}
\hat{\mathbf{x}}_{\text {in }} \\
\hat{\mathbf{x}}_{\text {out }}
\end{array}\right]=\mathbf{S}^{\dagger} \boldsymbol{\phi}
$$

where $\mathbf{S}^{\dagger}$ is the pseudoinverse of $\mathbf{S}$. Apparently, the condition number of $\mathbf{S}$ is high, yielding an unstable estimate $\hat{\mathbf{X}}$. However, this is mainly due to the different units of the harmonic expansion amplitudes, leading to highly different norms of the columns of $\mathbf{S}$. Thus, the basis $\mathbf{S}$ can be stabilized by normalizing the basis vectors, and in most practical applications with a multichannel device, $\hat{\mathbf{x}}$ can be calculated without any regularization. This is very convenient as regularization usually contains parameters that are difficult to determine optimally and because regularization leads to a biased estimate. After using a normalized basis in (8), one gets the true idealized channels simply by dividing the components of $\hat{\mathbf{x}}$ by the corresponding norms of the nonnormalized columns in matrix $\mathbf{S}$.

Calculation of (8) can be further stabilized by leaving out those components from the SSS basis that are dominated by noise. These components can be determined by examining the sensitivity of the sensor array to different harmonic functions. In some cases, the contribution of the harmonic components clearly falls below the noise level of the sensors, even if these components have values of $l$ smaller than the chosen highest order. Effectively, this selective adoption of basis vectors can be thought of as a regularization method based on physics and geometry.

\section{SUPPRESSION OF EXTERNAL INTERFERENCE SigNALS}

\section{A. Source Volumes of Biomagnetic Measurements}

With SSS utilizing spherical harmonic functions, the internal and external source volumes are defined by two spheres with radii $r_{\min }$ and $r_{\text {max }}$, respectively. For clarity, let us consider the case with common origin for the internal and external harmonic expansions. Then, $r_{\min }$ is the distance from the origin to the closest measurement point (see Fig. 6), and $r_{\max }$ is the distance from the origin to the farthest measurement point. All signals generated by sources with $r<r_{\min }$ are spanned by the subspace $\mathbf{S}_{\mathrm{in}}$, and all signals generated by sources with $r>r_{\max }$ are spanned by $\mathbf{S}_{\text {out }}$. Consequently, according to (8), the contribution of the internal and external sources can be separated from each other.

Sensor arrangements of the biomagnetic multichannel devices enable clear separation of the biomagnetic and external interference signals. The example in Fig. 6 illustrates a typical neuromagnetic measurement where the biomagnetic sources, i.e., the currents in the brain, are located in the volume with $r<r_{\min }$, and the external interference sources are located in the volume with $r>r_{\max }$. The SSS method is able to separate from each other the signals arising from sources in these two volumes.

\section{B. Software Magnetic Shield}

The SSS method suppresses the external interferences using minimal assumptions. However, as the method is based solely on the physics and geometry of the fields and the sensor array, it is sensitive to the accuracy of the calibration of the measurement device, in contrast to the SSP method [10], for example. In order to suppress the interference caused by nearby sources, a high-dimensional SSS basis must be used, which increases the noise sensitivity of the basis. Therefore, accurate calibration and 


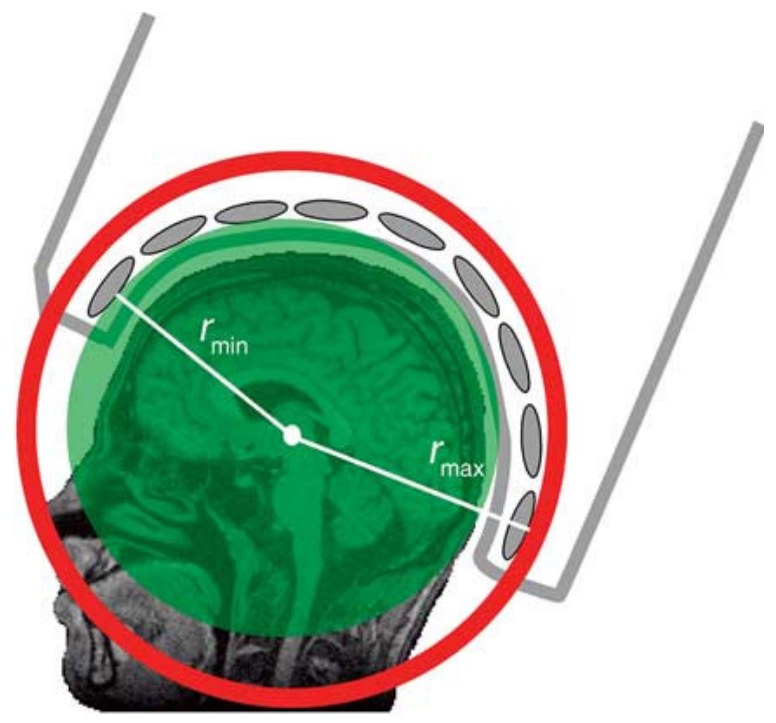

Fig. 6. Source volumes of a neuromagnetic measurement.

proper sensor configuration is necessary for optimal operation of SSS. On the other hand, because of its sensitivity to calibration, SSS can be used as a precise calibration method; deviations of the measured signals from the SSS model are due to calibration errors and can be used to correct the calibration parameters.

Increasing the dimension of the SSS basis refines the reconstruction but also increases the sensitivity of the SSS reconstruction to calibration errors. On the other hand, this sensitivity is also affected by the geometry of the sensor array. This is described by the ratio of the largest and smallest singular values of the SSS basis matrix $\mathbf{S}$, which is also called the condition number $\kappa$. The reconstruction error due to measurement errors and perturbations of the SSS basis matrix is proportional to $\kappa$ when numerical regularization is not used.

The level of software shielding achievable by SSS is studied here by simulating a source moving continuously from the interior to the exterior of the sensor array. The corresponding signal vector is decomposed in the SSS basis without numerical regularization, and the signal corresponding to the internal sources is reconstructed. The shielding factor is defined as $\|\phi\| /\left\|\mathbf{S}_{\text {in }} \hat{\mathbf{X}}_{\text {in }}\right\|$, which is the ratio of the norms of the actual signal vector and the reconstructed signal vector. Ideally, this factor would be 1 when the source is located inside the sensor array $\left(r_{\text {source }}<r_{\min }\right)$ and infinite when $r_{\text {source }}>r_{\max }$. In practice, the dominating magnetic interference contribution from a device inside of the magnetic shield is dipole by character. Consequently, we use in this simulation a dipolar source.

Fig. 7 shows the shielding factor of the SSS reconstruction with three different calibration accuracies for the case $L_{\text {in }}=$ $8, L_{\text {out }}=4$. For simplicity, random calibration errors were only produced for the scalar calibration coefficients and the balance of the gradiometer channels. The sensor array used in the simulation was the 306-channel Elekta Neuromag ${ }^{\circledR}$ MEG device including 204 planar gradiometers and 102 magnetometers. The imbalance signal was modeled as the signal of a point magnetometer located at the center between the gradiometer loops and with a normal in the plane of the gradiometer. The source was a magnetic dipole with dipole moment pointing to direc-

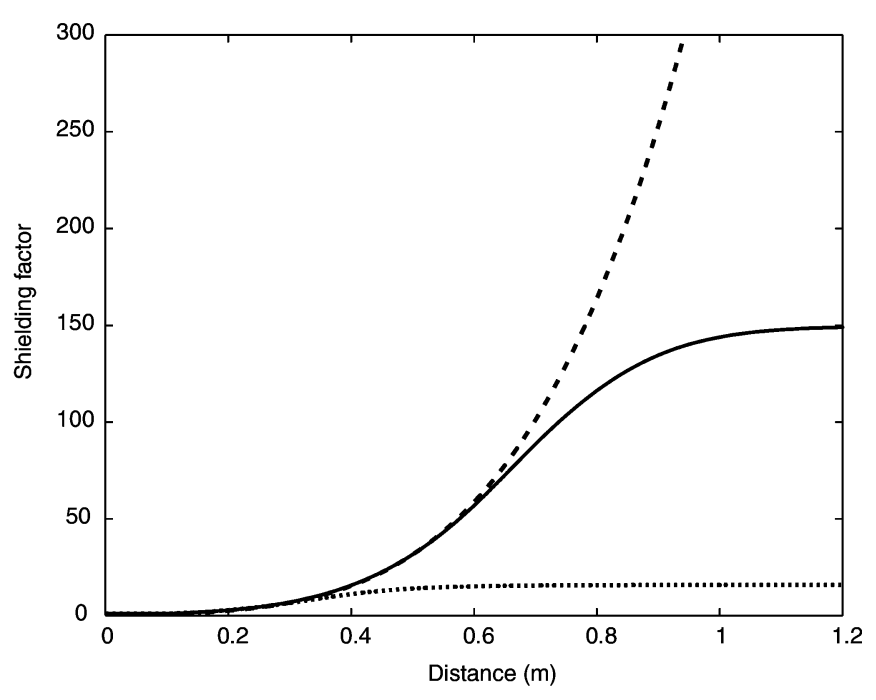

Fig. 7. SSS shielding factor as a function of distance of the source from the origin with three calibration accuracies. Upper (dashed) curve: Perfect accuracy. Middle curve: accuracy of $0.1 \%$. Lowermost curve: Accuracy of $1 \%$. The curves have been calculated with $L_{\text {in }}=8, L_{\text {out }}=4$.

tion $[1,1,1]$ moved along the negative $\mathrm{z}$-axis of the device coordinate system from 0 to $-3 \mathrm{~m}$, and the center of the harmonic expansions was at the origin of the device coordinate system. In this case, $r_{\min }=0.1063 \mathrm{~m}$, and $r_{\max }=0.1457 \mathrm{~m}$. It is seen that when using SSS, a "software shielding wall" forms in the region $r_{\text {source }}>r_{\text {min }}$. When the interference source moves further away through this wall, the shielding factor grows monotonously toward an asymptotic value limited by the calibration accuracy. A calibration accuracy of $0.1 \%$ gives an asymptotic shielding factor of about 150 , whereas this factor with calibration accuracy of $1 \%$ is about 15 only.

Fig. 8 demonstrates how the width of the software shielding wall is affected by the value of $L_{\text {out }}$ when $L_{\text {in }}=8$ and the calibration accuracy is $0.1 \%$. The asymptotic value of the shielding factor is higher for lower values of $L_{\mathrm{out}}$, but a shielding wall closer to the helmet is achieved by increasing $L_{\text {out }}$. The latter is desirable for interference sources located very near the sensor array. From Fig. 8, one can actually determine which value of $L_{\text {out }}$ is optimal for an interference source at a given distance. For example, in the range $0.7 \mathrm{~m}<r_{\text {source }}<1.2 \mathrm{~m}, L_{\text {out }}=4$ would be optimal.

The effect of the SSS-operation on the noise depends crucially on the geometry of the sensor array, as represented by the condition number of the SSS basis. These numbers for three different whole head MEG devices, 306 channel (Elekta Neuromag ${ }^{\circledR}$ triple sensors), 275 channel (5-cm base axial gradiometers), and 248 channel (magnetometers) relate to each other as 1:1.26:1.37 when $L_{\text {in }}=8, L_{\text {out }}=4$ are used. For these parameter values, there is on the average no increase in the noise for the 306-channel system. The better condition number of the 306channel device stems from the intrinsic differentiation between nearby and far-away sources achieved by the triple sensor comprising both short base-length gradiometers and magnetometers.

The above simulations show that with a properly designed sensor array utilizing SSS, it is possible to reach shielding factors exceeding 100 even for interference sources closer than 1 


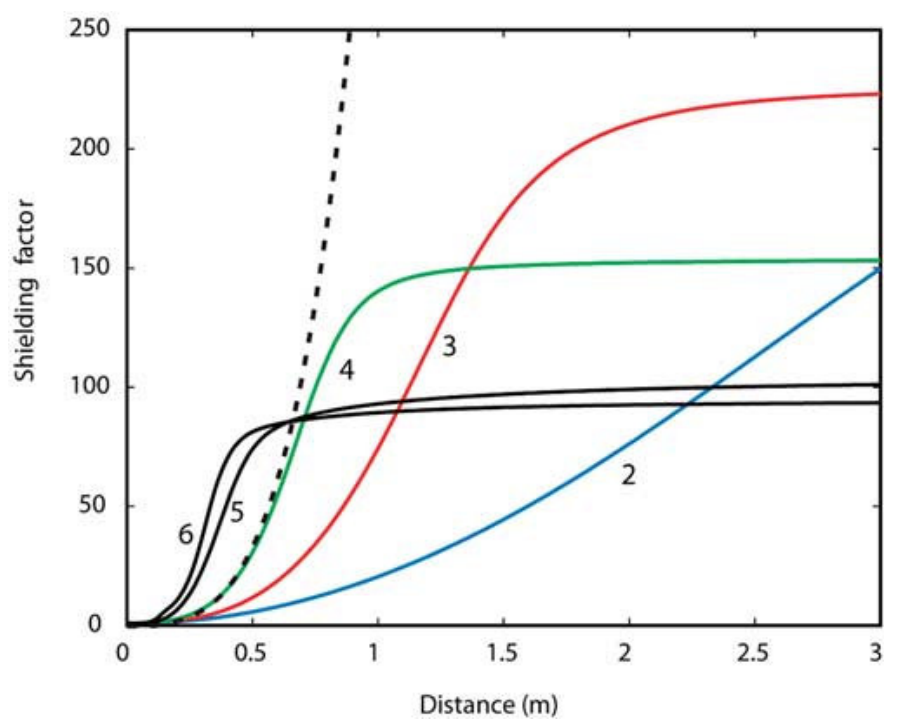

Fig. 8. SSS shielding factor as a function of distance of the source from the origin with five different values of $L_{\text {out }}$ with $L_{\text {in }}=8$. The $L_{\text {out }}$ values for curves from left to right are $6,5,4,3$, and 2 . The curves correspond to calibration accuracy of $0.1 \%$. The dashed curve corresponds to perfect calibration in the $L_{\text {out }}=4$ case

$m$ to the device. Importantly, while suppressing the interference, the morphology of the brain signals is not affected in SSS, which is in contrast to methods that completely remove some particular temporal or spatial patterns that are assumed to only contain interference. Such methods achieve arbitrarily high shielding factors against the predefined interference patterns with the cost of removing the projection of the brain signal to these patterns. In principle, external interference suppression using reference channels leaves the morphology of the brain signals intact, provided that the assumption of the reference sensors only measuring interference is valid. For this assumption to hold, the reference sensors have to be located at a sufficiently large distance from the signal sensors. On the other hand, the large distance reduces the accuracy of the interference extrapolation. Consequently, the shielding factor of a practical reference array is reported to be on the order of 20 for far-away interference sources [9]. For interference sources at a distance of $1 \mathrm{~m}$, it is even considerably less. SSS provides a more efficient interference suppression method as it does not need to distinguish between signal and reference channels or extrapolate signals from any sensor to any other. All channels give information of both the brain signals and external interference that can be separated from each other with the simple procedure described in Section II.

\section{Experimental Results on Software Magnetic Shielding}

The simulation of the previous section was verified experimentally. For practical reasons, we moved the magnetic dipole inside of a magnetically shielded room from $0 \mathrm{~m}$ to $-1.93 \mathrm{~m}$ only. Apart from this, the parameters of the simulation were repeated as accurately as possible in the measurement. Fig. 9 shows for $L_{\text {in }}=8$ and $L_{\text {out }}=4$ the measured shielding factor of the SSS reconstruction with the "as received" calibration accuracy of about $1 \%$ and with the SSS-based fine calibration accuracy of about $0.15 \%$ achieved at that time. The results closely

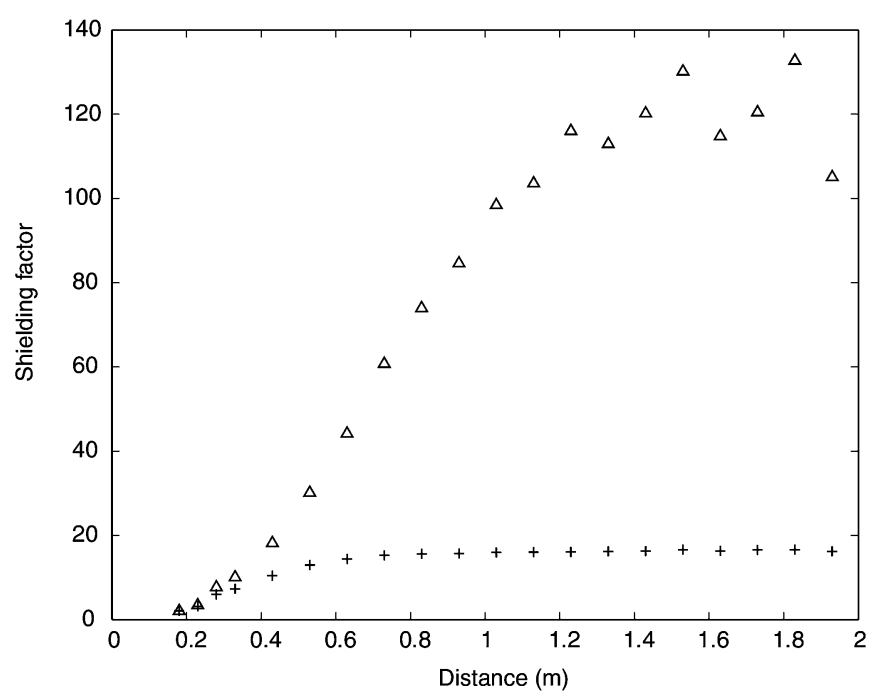

Fig. 9. Measured SSS shielding factor with a fine calibration accuracy of $0.15 \%$ (upper curve) and conventional "as received" accuracy of approximately $1 \%$ (lower curve).

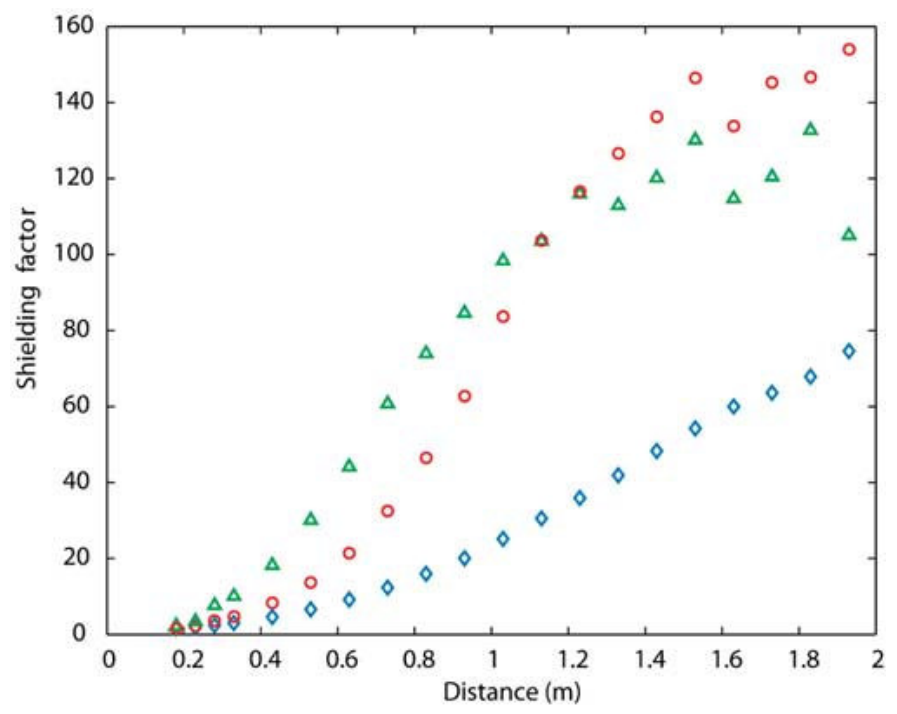

Fig. 10. Measured SSS shielding factor with three different values of $L_{\text {out }}$ with $L_{\text {in }}=8$ and with fine calibration. Triangle corresponds to $L_{\text {out }}=4$, circle corresponds to $L_{\text {out }}=3$, and diamond corresponds to $L_{\text {out }}=2$.

follow the simulation results of Fig. 7. Fig. 10 shows the measured shielding factor with fine calibration for $L_{\text {out }}$ values of 2 , 3 , and 4 when $L_{\text {in }}=8$.

Fig. 11 demonstrates suppression of external interference signals from an empty room measurement. In this case, we know that there are no biomagnetic signals, and thus, the SSS reconstructed signal should be zero. However, as anticipated, SSS is able to suppress the interference only by a factor of 15-20 with the "as received" calibration accuracy. On the other hand, SSS with fine calibration gives interference suppression by a factor exceeding 200, as seen by comparing the top and bottom traces in Fig. 11.

To demonstrate that SSS suppresses the external interference signals without affecting the biomagnetic signals, we placed a phantom head containing artificial current dipoles inside the 

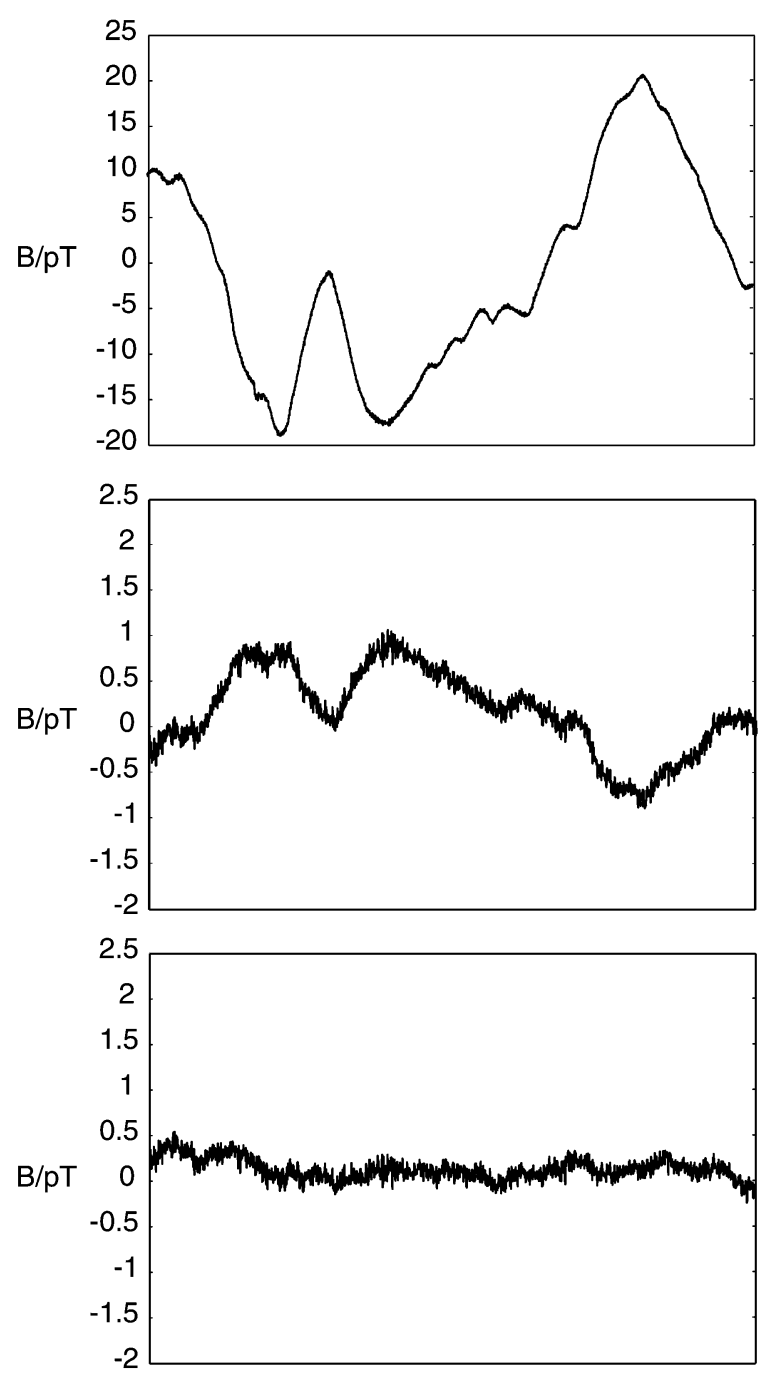

$20 \mathrm{~s}$

Fig. 11. Time trace of one magnetometer channel from an empty room measurement. (Top) Original signal. (Middle) SSS reconstructed signal with "as received" calibration. (Bottom) SSS reconstructed signal with fine calibration. Note that the scale in the top figure is by a factor 10 larger than in the other two figures.

sensor array. Fig. 12 shows time traces of two orthogonal gradiometers and a magnetometer in one triple sensor of Elekta Neuromag ${ }^{\circledR}$. In this case, the external interference signals were obviously generated by sources located far away from the sensors. Thus, the original gradiometer signals do not contain interference but only the sine wave burst produced by the phantom dipole. The SSS reconstruction leaves the gradiometer signals and their noise level essentially intact. On the other hand, the magnetometer channel suffers from low-frequency interference, which is removed by SSS without affecting the phantom signal.

Figs. 13 and 14 demonstrate SSS reconstruction of a single, unaveraged auditory response of a newborn baby. In this experiment, the baby had her left hemisphere leaning against the back of the helmet, and we expect to see the auditory response in the middle of the sensor group shown in Fig. 14. However, a heartbeat of the baby partially obscured the brain response, as can be seen in the original waveforms and field maps. In Fig. 14, the original field map is distorted by the heart signal, but the

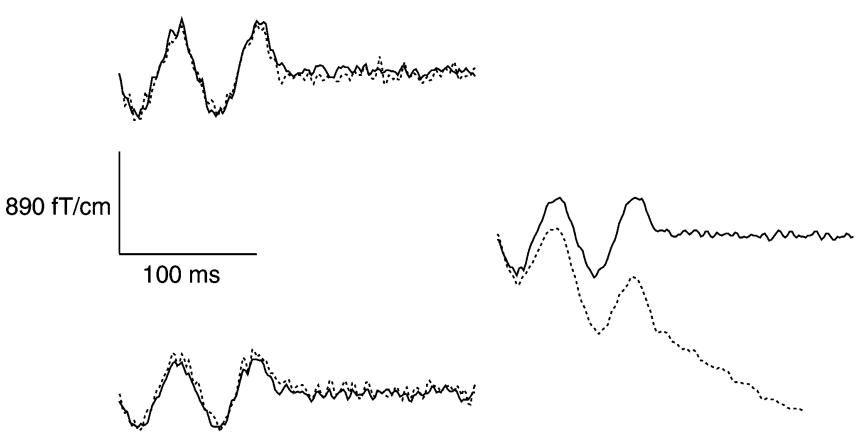

Fig. 12. Original (dashed) and SSS reconstructed (solid) unaveraged signals from three independent channels of one triple sensor in a phantom measurement. The two gradiometers are on the left side, and the magnetometer is on the right side.

dipolar auditory signal is also visible. Dipolarity of the field becomes clearer in the SSS reconstruction, and good suppression of the hearbeat requires a high $L_{\text {out }}$ because the heart of the newborn is located very close to the sensor array. Figs. 13 and 14 indicate that $L_{\text {out }}=5$ is sufficient for suppression of this interference source that is very close to the helmet.

\section{Standardization Between DifFerent MEASUREMENT CONFIGURATIONS}

\section{A. Alignment of Different Head Positions}

Different locations of the subjects with respect to the measurement device hamper the comparison of data from different measurement sessions. In order to remove this uncertainty, the signals have to be aligned to correspond to a standard head position. The recipe for the conversion is simple: Transform the measured signals into a device-independent representation attached to the coordinate system of the head, and calculate the virtual signals that would have been measured from a standard head position. Suitable device-independent source models to be used for virtual signals are, e.g., MNE [14] and multipole expansion [15]. Our implementation of SSS uses the multipole expansion and enables virtual signal calculation with the advantage that the multipole components are first calculated for both the biomagnetic and external interference signals. Then, the virtual signals can be calculated from unbiased multipole components corresponding to the biomagnetic signals only. The basic principle is explained in detail in [17].

To demonstrate the ability of SSS to align different head positions, we measured and averaged the auditory response of a subject from two head positions and then converted the measurements to a standard head position. In the first measurement, the subject was leaning against the left side of the inside surface of the Elekta Neuromag ${ }^{\circledR}$ helmet, and in the second measurement, he leaned against the right inside surface of the helmet. The original unprocessed waveforms are shown overlayed on the left side of Fig. 15, and the morphology of the response is clearly altered by the different head positions. Furthermore, both measurements suffer from a low-frequency interference dominating the magnetometer signal amplitudes because no interference compensation was applied during data acquisition. The right side of Fig. 15 shows the standardized signals from both measurements overlayed. These signals are the virtual signals 

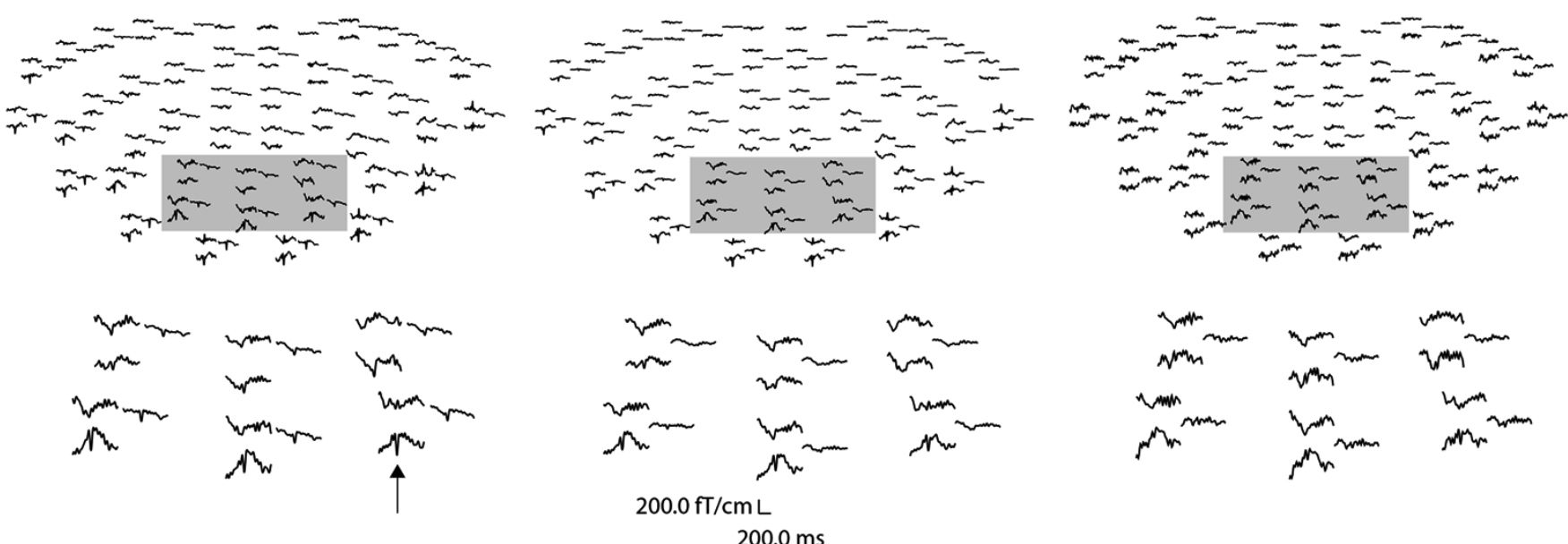

Fig. 13. Channel layouts representing a single trial auditory response of a newborn baby. (Left) Original signals contaminated by a heartbeat. (Middle) SSS reconstructed signals with $L_{\text {in }}=8, L_{\text {out }}=3$. (Right) SSS reconstructed signals with $L_{\text {in }}=8, L_{\text {out }}=5$.
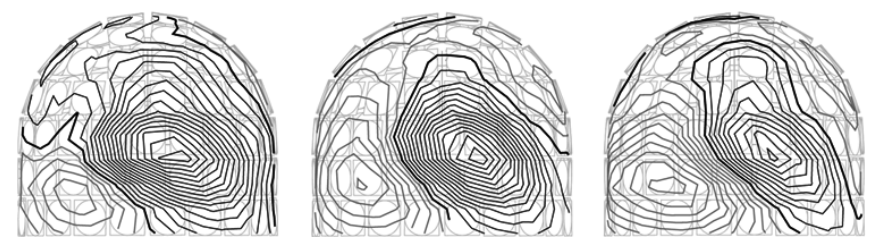

Fig. 14. Field maps from a single auditory response of a newborn baby. (Left) Original field (Middle) SSS reconstructed field with $L_{\text {in }}=8, L_{\text {out }}=3$. (Right) SSS reconstructed field with $L_{\text {in }}=8, L_{\text {out }}=5$.

that would have been measured if the subject would have had the center of his head $2.3 \mathrm{~cm}$ above the centers of the lowest sensors in the sensor array and with the coordinate axes of the head aligned with the coordinate axes of the device. In this case, the standardization generally increases the signals because the subject was located lower in the real measurement than in the standardized result. The standardized signals from both measurements are in good accordance with each other and show that SSS suppresses the discrepancy caused by the different head positions. Furthermore, the external interference is automatically removed in the SSS conversion.

\section{B. Comparison of Data Acquired With Different Sensor Arrays}

In addition to the discrepancy caused by different head positions, differences between measurement devices may prevent comparison of MEG data. However, with SSS, one can easily transform measurements between different biomagnetic instruments by estimating the harmonic amplitudes from the real measurement and calculating the virtual signals using this estimate and the internal SSS basis $\mathbf{S}_{\text {in }}$ of the instrument whose signals in which one is interested. Mathematically, this operation is equivalent to standardizing different head positions discussed in the previous section. As long as the device used for the real measurement is capable of recording data with enough information content (for a definition, see e.g., [23]), the harmonic amplitudes can be reliably estimated, and the measurement can be transformed to any sensor configuration. This facilitates comparison between results obtained by different researchers using different instruments.
Our practical example shown in Fig. 16 [24] is from an averaged MCG measurement where the heart signals were measured with a 99-channel cardiomagnetometer at the BioMag Laboratory of the Helsinki University Central Hospital (HUCH) and then converted using SSS into 49 axial gradiometer signals corresponding to the device at Physikalisch-Technische Bundesanstalt (PTB), Berlin, Germany. Fig. 16 shows the measured signals converted from BioMag's device to PTB's device, along with the signals actually measured at PTB from the same subject. In this case, orderds $L_{\text {in }}=4$ and $L_{\text {out }}=2$ were used. The figure shows very good agreement between the converted and measured data. The amplitude-weighted correlation used as a measure of reproduction accuracy in [15] was 0.980 in this case.

\section{MOVEMENT COMPENSATION}

\section{A. Movement Distortions}

Movement of the subject during the recording may severely distort MEG data. With cooperative healthy subjects, this is not a problem, but, e.g., with small children and some patient groups, head movements are unavoidable. Even with very large movement, the recorded MEG signals from a focal source often still appear dipolar, although they are blurred by the movement. This is a potential risk as the source modeling of such data may lead to incorrect localization with statistically high confidence. In such a case, the only reliable indication of the head movement is the result of a continuous head position monitoring system. The analysis of such data requires movement compensation, which consists of dynamic continuous recording of the head position and a method that takes the recorded movement into account in the analysis.

Movement compensation requires decomposing the measured signals into a source model attached to the coordinate system of the head. One possible source model is the minimum norm estimate. However, SSS provides a better source model for this purpose as it also automatically suppresses the external interference signals [17] and is computationally fast.

By modeling the movement of the subject as movement of the sensor array, the subject can be considered static in terms of the decomposed biomagnetic field components. Thus, virtual 

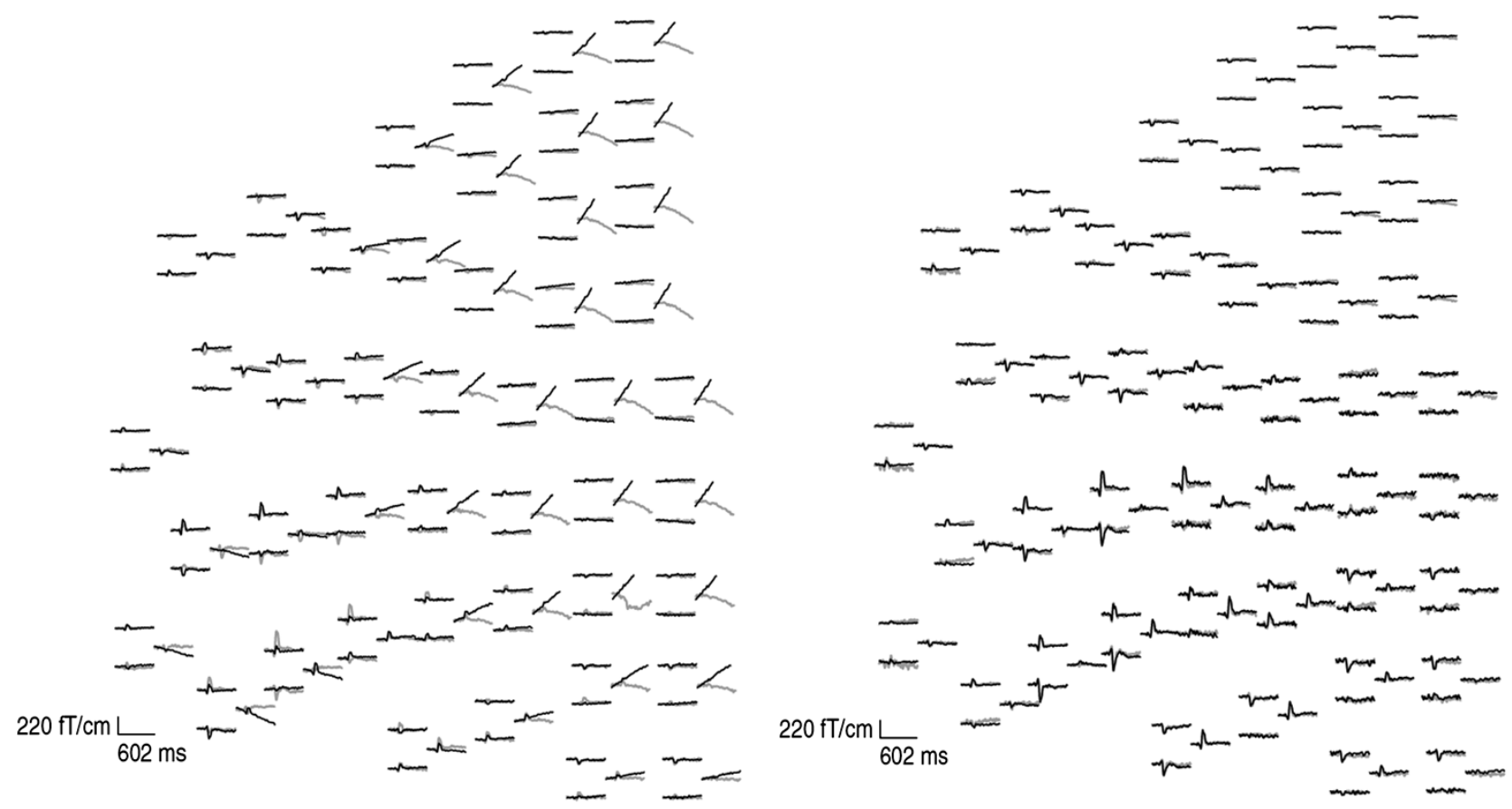

Fig. 15. Auditory responses at the left frontal quadrant of the sensor array. (Left) Original waveforms of an auditory response recorded from two different head positions with no interference compensation. Grey and black traces correspond to subject leaning to the left and to the right, respectively. (Right) Standardized waveforms of an auditory response recorded from two different head positions. Grey and black traces correspond to standardizations made for subject leaning to the left and to the right, respectively. Interference has been automatically compensated for in the SSS process.

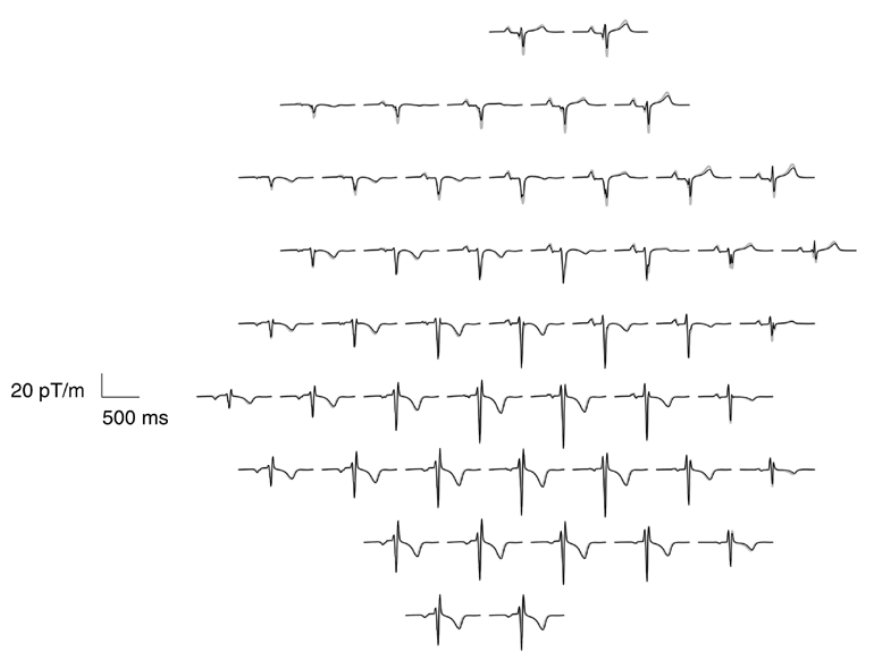

Fig. 16. Comparison of converted and measured axial gradiometer signals. The grey signals represent the 99-channel data from BioMag converted to the channel layout of PTB. The black signals represent an actual measurement of the same subject at PTB (published with permission of PTB).

signals corresponding to any reference head position can be calculated based on the estimated components, and these movement-compensated signals can be used for further data analysis, as if the subject had not moved during the measurement.

Our practical demonstration of Fig. 17 is from an auditory measurement. An auditory response was measured from a subject continuously moving his head in a range of about $5 \mathrm{~cm}$. The resulting field appears dipolar but is convolved by the movement and gives a localization error of $41 \mathrm{~mm}$, as compared to the reference response with no head movements. SSS deconvolves the data and the resulting localization agrees within $3 \mathrm{~mm}$ with
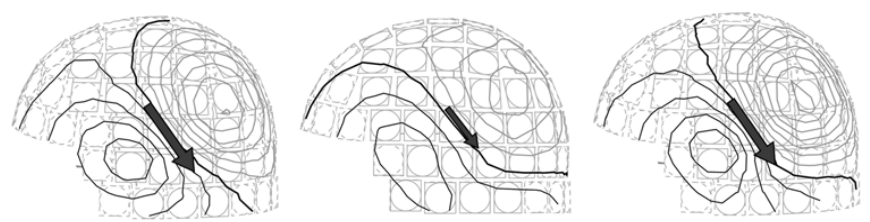

Fig. 17. Auditory response from (left) a stable subject,(middle) from a moving subject without movement correction, and (right) from a moving subject after SSS-based movement correction.

that of the reference response from stable measurement from the same subject. The continuous head position monitoring was based on the method described in [13].

\section{B. Artifacts Caused by Magnetic Impurities}

Magnetic impurities on the head or body are DC sources that, when stationary, do not produce signals in MEG measurement as the SQUID sensors are only sensitive to fields that are dynamic in their coordinate system. However, if the subject moves with respect to the device, these DC signals will be modulated by the movement and appear as artifact signals. Such artifacts can be encountered, e.g., with patients having involuntary movements and containing tiny magnetic particles left from surgical drills, for example. These artifact signals are often very strong as compared with the brain responses and typically lead to rejection of the data from further analysis. Fortunately, with SSS-based movement compensation, the movement-modulated DC signals will appear as static components in the reconstructed signals and can be removed with a simple baseline correction. Recovery of an SEF-response buried under this kind of movement artifact that is much stronger than the brain response has been demonstrated in [16]. 


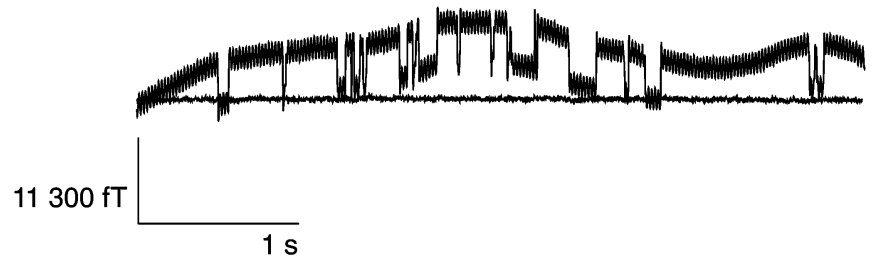

Fig. 18. Original and SSS reconstructed signal of one misbehaving magnetometer channel showing a step-like artifact signal and power line interference. The signal is from an empty room measurement.

\section{OTHER APPLICATIONS}

In Sections III-V, we have described the solutions for several problems in biomagnetic measurements by means of SSS. In this section, we list other specific applications of SSS that can significantly improve the quality of biomagnetic signals and data analysis.

SSS suppresses possible sporadic sensor artifacts of electronics origin, for example. These artifact signals correspond to sources in the sensor volume and, thus, are not modeled by the SSS basis. As there is usually only a small number of malfunctioning channels, if any, and since their artifact signals are usually uncorrelated, the sensor artifacts correspond to signal vectors that are approximately orthogonal to the SSS basis when the number of channels is high. Consequently, the contribution of the sensor artifacts is suppressed by a large factor in the SSS reconstruction, as is the case with any reasonable source model (see Fig. 18).

In addition to suppressing distortions and artifacts, SSS provides a straightforward tool for phantom-free fine calibration that is capable of utilizing any magnetic signals, for example, external interference. With the assumption that all interference sources are located more than a few of centimeters from the sensors, any deviations of the measured signal vectors from the SSS subspace must be caused by inaccurate knowledge of the geometry of the sensor array and gains of the channels. By fine adjusting these calibration parameters in such a way that all the signals fall into the calculated SSS basis, the system becomes consistent with Maxwell's equations. This provides a more accurate and robust calibration method than the traditional way of using a predesigned probe, e.g., a phantom head, because in the latter method, the imprecise knowledge on the geometry of the phantom is also a source of calibration errors. Our method only requires that the calibrating field obeys Maxwell's equations, which is a condition that is obviously fulfilled by any magnetic field. In practice, this SSS-based calibration requires that the sensors can be well characterized by a small number of parameters, and it is, therefore, especially suited for calibration of thin film devices having excellent geometric precision and reproducibility.

In addition to removing movement artifacts caused by magnetic particles, the SSS basis can also be utilized in extracting physiological DC components. This can be accomplished, e.g., by recording signal variations as a function of time and by reconstructing the signals into idealized channels corresponding to the coordinate system of the head. Dynamic brain signals are uncorrelated with the head movements, whereas the DC currents inside the moving head produce a time-varying signal that can be completely described by the movement. Thus, the movement-modulated signal produced by the DC currents can be demodulated back to DC by utilizing continuous head position monitoring and SSS, as shown in [25].

The idealized channels $\alpha_{l m}$, as defined in (1), represent the measured data in a compact form, which is more tractable in terms of source modeling than the outputs of real channels. For example, a current dipole in the spherical model can be localized analytically from the multipole components. The Appendix shows an analytic solution utilizing a subset of the multipole moments. Calculation of distributed source models is also facilitated by SSS [17].

SSS enables a straightforward and robust realization of a feedback-based active compensation system utilizing actual signal channels as zero detectors driving compensation coils inside the shielded room [26]. The morphology of the biomagnetic signals is not altered in the compensation process because SSS suppresses the signals of the compensation coils that represent external interference sources. Making the passive magnetic shielding considerably lighter than in the traditional two to three layer magnetically shielded rooms is thus considerably facilitated.

\section{CONCLUSION}

We have demonstrated that SSS idealizes biomagnetic multichannel measurements by means of a simple linear model. The idealized signals correspond to a measurement of a nonmoving subject in an environment with no external interference signals. Furthermore, the decomposition can be represented in a form of idealized channels containing uncorrelated information. Thus, SSS relaxes many of the traditional restrictions and limitations of biomagnetic measurements.

The linear SSS basis relies on Maxwell's equations and the geometry of the sensor array. The only additional assumption is that all sources-both biomagnetic and interference sources-are located more than a couple of centimeters from the sensors. Since this assumption is generally valid in biomagnetism, all multichannels signals of magnetic origin are spanned by the SSS basis with separate basis vectors for signals arising from inside of the sensor array, the biomagnetic sources, and for signals arising from outside of the sensor array, i.e., the external interference sources.

The software magnetic shielding provided by the SSS method contributes especially to clinical applicability of MEG. Normal working practices and stimulators of a neurological clinic can even be applied inside of the magnetic shield without extra attention and training of the personnel, regarding sources of magnetic interference. A high-precision multichannel MEG device calibrated to a relative accuracy of about $0.1 \%$ or better is a necessary prerequisite for efficient magnetic shielding based on SSS.

The idealized channels provided by SSS contain the measured biomagnetic information in a compact form. The signals of these channels can be used for an efficient visualization of the data as the number of ideal channels is much smaller than the number of real channels. Furthermore, virtual signals corresponding to any desired sensor configuration can be easily 
calculated from the idealized channels. This can be used, e.g., for standardizing head positions or transforming data from one measurement device to another.

Movement of the subject may severely distort MEG data without completely destroying the typical spatial pattern of high-quality MEG data. Source modeling of such distorted data generally leads to an erroneous localization result. This is especially harmful in clinical work where accurate localization is crucial and the patients cannot be expected to stay still during the measurement. Consequently, a continuous movement-detection system and a mathematical movement-compensation method are important for reliable MEG analysis. SSS provides an accurate and efficient method to compensate for movements in MEG. It also enables movement artifacts caused by magnetic impurities to be removed by a simple baseline correction.

Other applications of SSS include fine calibration, recording of physiological DC phenomena, suppression of sporadic sensor artifacts, facilitated source modeling, and active compensation utilizing compensation coils inside the shielded room.

\section{APPENDIX}

\section{ANALYTICAL LOCALIZATION OF A CURRENT DIPOLE}

We derive analytical expressions for localization of a current dipole in the spherically symmetric conductor model. The following solution only contains low-order multipole components and is not statistically optimal in the sense of maximum likelihood estimators, but it is computationally fast. Furthermore, the analytical solution can be used as a good initial guess for nonlinear search of the dipole.

The current dipole $\mathbf{Q}$ can be thought of as a concentration of the primary current to a single point. Because the radial part of the dipole does not produce a magnetic field outside the volume conductor, we can express the dipole using only the tangential unit vectors

$$
\mathbf{Q}=Q_{\theta} \mathbf{e}_{\theta}+Q_{\varphi} \mathbf{e}_{\varphi}
$$

Let us express the scalar potential $V_{\text {in }}(\mathbf{r})$ corresponding to the biomagnetic sources in the real form [27]

$$
V_{\mathrm{in}}(\mathbf{r})=\frac{1}{4 \pi} \operatorname{Re} \sum_{l=1}^{\infty} \sum_{m=0}^{l} M_{l m} \frac{\tilde{Y}_{l m}^{*}(\theta, \varphi)}{r^{l+1}}
$$

where $\tilde{Y}_{l m}(\theta, \varphi)=P_{l m}(\cos \theta) e^{\mathrm{i} m \varphi}$ is the non-normalized spherical harmonic function, and $*$ denotes complex conjugate. The coefficients are given as a function of the total current distribution $\mathbf{J}\left(\mathbf{r}^{\prime}\right)$ by

$$
M_{l m}=-\frac{\xi_{l m}}{l+1} \int_{v^{\prime}} r^{\prime l} \tilde{Y}_{l m}\left(\theta^{\prime}, \varphi^{\prime}\right) \nabla^{\prime} \cdot\left(\mathbf{r}^{\prime} \times \mathbf{J}\left(\mathbf{r}^{\prime}\right)\right) d v^{\prime}
$$

where

$$
\xi_{l m}=\left(2-\delta_{m 0}\right) \frac{(l-m) !}{(l+m) !}
$$

and $\delta_{i j}$ is Dirac's delta function. Let us denote

$$
\chi_{l m}=\operatorname{Re}\left(M_{l m}\right)
$$

and

$$
\tau_{l m}=\operatorname{Im}\left(M_{l m}\right)
$$

giving us, according to (10)

$$
\begin{aligned}
V_{\text {in }}(\mathbf{r})=\frac{1}{4 \pi} \sum_{l=1}^{\infty} \sum_{m=0}^{l} \frac{P_{l m}(\cos \theta)}{r^{l+1}} \\
\times\left[\chi_{l m} \cos (m \varphi)+\tau_{l m} \sin (m \varphi)\right]
\end{aligned}
$$

which is compatible with the usual expression using even and odd multipoles; see, e.g., [19].

In order to calculate analytical expressions for localizing a current dipole, one can utilize the expressions giving the harmonic amplitudes as a function of the current distribution. For example, by comparing the lead field form of the idealized channels $\alpha_{l m}$ with (11), or by using the amplitude expressions in [19], we can find the analytical equations for localization of the dipole.

Let us first define

$$
\begin{aligned}
\tilde{\chi}_{i j} & =\chi_{i 0} \chi_{j 0} \\
\rho & =\chi_{11}^{2}+\tau_{11}^{2} \\
\gamma & =15 \chi_{20}^{2}-16 \tilde{\chi}_{13} \\
\eta & =3 \chi_{20}^{2}-4 \tilde{\chi}_{13} .
\end{aligned}
$$

Then, we get the following analytical expressions for the spherical coordinates and moment of the dipole:

$$
\begin{aligned}
r_{\text {dip }} & = \pm \frac{1}{2 \sqrt{3}} \frac{\sqrt{\gamma}}{\chi_{10}} \\
\theta_{\text {dip }} & =\arccos \left( \pm \frac{\chi_{20} \sqrt{3}}{\sqrt{\gamma}}\right) \\
\varphi_{\text {dip }} & =\arcsin \left(\frac{1}{2 \rho}\left[\chi_{11} \sqrt{4 \rho-\frac{3 \tilde{\chi}_{12}^{2}}{\eta}} \mp \frac{\sqrt{3} \tilde{\chi}_{12} \tau_{11}}{\sqrt{\eta}}\right]\right) \\
Q_{\theta} & = \pm 2 \sqrt{3} \frac{\chi_{10}}{\sqrt{\gamma}} \sqrt{4 \rho-\frac{3 \tilde{\chi}_{12}^{2}}{\eta}} \\
Q_{\varphi} & =\mp \frac{\chi_{10}^{2} 2 \sqrt{3}}{\sqrt{\eta}} .
\end{aligned}
$$

The signs are chosen in such a way that the results are consistent with the multipole coefficientes $\chi_{l m}$ and $\tau_{l m}$.

\section{ACKNOWLEDGMENT}

The authors acknowledge L. Parkkonen for Fig. 6, V. Mäntynen for Fig. 16, and PTB for the permission to use their data. The authors would like to thank Prof. P. Kuhl, Prof. T. Imada, and Prof. M. Cheour for providing us with their infant MEG data, which acted as a crucial stimulation for the development of the SSS method. The authors also thank Dr. A. Ahonen for very useful comments on the manuscript and Dr. J. Nenonen for providing them with some important numerical calculations used in this publication. 


\section{REFERENCES}

[1] M. Hämäläinen, R. Hari, R. J. Ilmoniemi, J. Knuutila, and O. V Lounasmaa, "Magnetoencephalography-Theory, instrumentation, and applications to noninvasive studies of the working human brain," Rev Mod. Phys., vol. 65, Apr. 1993.

[2] The SOUID Handbook: Volume 1: Fundamentals and Technology of SQUID's and SQUID Systems, Wiley, New York, 2004. J. Clarke and A. Barginski.

[3] M. Pannetier, C. Fermon, G. L. Goff, J. Simola, and E. Kerr, "Femtotesla magnetic field measurement with magnetoresistive sensors," Science, vol. 304, pp. 1648-1650, 2004.

[4] I. Kominis, T. Kornack, J. Allred, and M. Romalis, "A subfemtotesla multichannel atomic magnetometer," Nature, vol. 422, pp. 596-599, 2003.

[5] D. Cohen, "Large-volume conventional magnetic shields," Rev. Phys. Appl., vol. 5, pp. 53-58, 1970.

[6] V. Kelhä, J. Pukki, R. Peltonen, A. Penttinen, R. Ilmoniemi, and J. Heino, "Design, construction, and performance of a large-volume magnetic shield," IEEE Trans. Magn., vol. MAG-18, pp. 260-270, Jan. 1982

[7] J. Bork, H.-D. Hahlbohm, R. Klein, and A. Schnabel, "The 8-layered magnetically shielded room of the PTB: Design and construction," in Proc. Biomag, 2001, pp. 970-973.

[8] J. E. Zimmerman, "SQUID instruments and shielding for low-level magnetic measurements," J. Appl. Phys., vol. 48, pp. 702-710, 1977.

[9] J. Vrba and S. E. Robinson, "Signal processing in magnetoencephalography," Methods, vol. 25, pp. 249-271, 2001.

[10] M. A. Uusitalo and R. J. Ilmoniemi, "Signal-space projection method," Med. Biol. Eng., vol. 32, pp. 35-42, 1997.

[11] M. S. Hämäläinen and R. J. Ilmoniemi, "Interpreting Measured Magnetic Fields of the Brain: Estimates of Current Distributions," Helsinki Univ. Technol., Helsinki, Finland, Tech. Rep. TKK-F-A559, 1984

[12] J. Sarvas, "Basic mathematical and electromagnetic concepts of the biomagnetic inverse problems," Phys. Med. Biol., vol. 32, pp. 11-22, 1987.

[13] K. Uutela, S. Taulu, and M. Hämäläinen, "Detecting and correcting for head movements in neuromagnetic measurements," NeuroImag., vol. 14 pp. 1424-1431, 2001.

[14] J. Numminen, S. Ahlfors, R. Ilmoniemi, J. Montonen, and J. Nenonen, "Transformation of multichannel magnetocardiographic signals to standard grid form," IEEE Trans. Biomed. Eng., vol. 42, no. 1, pp. 72-78, Jan. 1995.

[15] M. Burghoff, J. Nenonen, L. Trahms, and T. Katila, "Conversion of mag netocardiographic recordins between two different multichannel SQUID devices," IEEE Trans. Biomed. Eng., vol. 47, no. 7, pp. 869-875, Jul. 2000.

[16] S. Taulu, M. Kajola, and J. Simola, "Suppression of interference and artifacts by the signal space separation method," Brain Topograph., vol. 16, pp. 269-275, 2004.

[17] S. Taulu and M. Kajola, "Presentation of electromagnetic multichannel data: The signal space separation method," J. Appl. Phys., vol. 97, Jun. 2005.

[18] J. D. Jackson, Classical Electrodynamics, Third ed. New York: Wiley, 1999

[19] J. P. Wikswo, Jr. and K. R. Swinney, "A comparison of scalar multipole expansions,” J. Appl. Phys., vol. 56, pp. 3039-3049, 1984.

[20] G. H. Golub and C. F. Van Loan, Matrix Computations, Third ed. Baltimore, MD: Johns Hopkins Univ. Press, 1996.

[21] E. L. Hill, "The theory of vector spherical harmonics," Amer. J. Phys., vol. 22 , pp. 211-214, 1954
[22] G. Arfken, Mathematical Methods for Physicists, Third ed. New York: Academic, 1985.

[23] J. Nenonen, M. Kajola, and A. Ahonen, "Total information of multichannel MEG arrays," in Proc. Biomag., 2004, pp. 630-631.

[24] V. Mäntynen, M. Karvonen, S. Taulu, J. Nenonen, J. Simola, M. Kajola, A. Ahonen, and T. Katila, "Signal space separation in magnetocardiography," in Proc. Biomag., 2004, p. 386.

[25] S. Taulu, J. Simola, and M. Kajola, "MEG recordings of DC fields using the signal space separation method (SSS)," in Proc. Biomag., 2004, pp. 184-185.

[26] J. Simola, S. Taulu, L. Parkkonen, and M. Kajola, "Active shielding method for an MEG device," in Proc. Biomag., 2004, p. 661.

[27] K. Jerbi, J. C. Mosher, S. Baillet, and R. M. Leahy, "On MEG forward modeling using multipolar expansions," Phys. Med. Biol., vol. 47, pp. $523-555,2002$

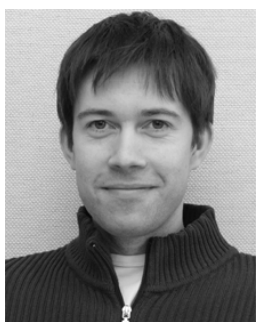

Samu Taulu was born in 1974 . He received the M.Sc. degree in physics from the Helsinki University of Technology (HUT), Helsinki, Finland, in 2000 where he is currently working toward the Ph.D. degree.

He joined the Low Temperature Laboratory, HUT, in 1997 and Neuromag Oy, Helsinki, in 1998. During the last few years, he has been developing mathematical methods for signal processing of electromagnetic multichannel data at Elekta Neuromag Oy.

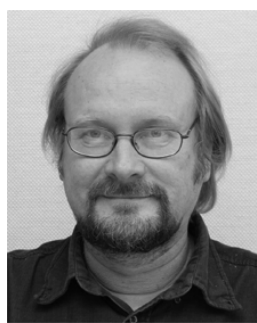

Juha Simola was born in 1952. He graduated in theoretical physics from Helsinki University of Technology (HUT), Helsinki, Finland, from which he received the Ph.D. degree in 1985. His dissertation was on quantized vortices in rotating $3 \mathrm{He}$ superfluids.

$\mathrm{He}$ is currently developing methods and instrumentation for clinical MEG at Elekta Neuromag Oy, Helsinki.

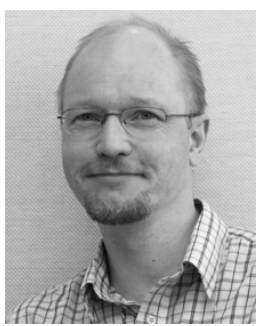

Matti Kajola was born in 1960 . He graduated in physics from Helsinki University of Technology (HUT), Helsinki, Finland, in 1986.

He joined the Low Temperature Laboratory, HUT, in 1983. He has been working on both hardware and software for whole-cortex neuromagnetometers. He is currently the head of the software department of Elekta Neuromag Oy, Helsinki. 